\title{
Game Platform Prototype for Understanding Safety Issues of Life Boat Launching Process
}

\begin{abstract}
The novel advanced game techniques provide us with new possibilities to mimic a complicated training process, with the benefit of safety enhancement and cost effective. In this paper, we design and implement a 3D game which imitates the lifeboat launching process. Lifeboat launching is such a complex but vital process which can on one side saving people's life on sea and on the other side associating many potential hazards. It involves both the tractor manoeuvres and boat operations. The primary objective of the game is allows novices to better understand the sequence of the operations in launching process and manager the potential hazards happening during the launching. There is also great educational significance with the promotion of the game to the general public for enhanced awareness of safety issues. The key modules of the game are designed based on physical simulation which gives the players enhanced plausible cognition and enjoyable interaction.
\end{abstract}

\section{INTRODUCTION}

Lifeboat launching is such a complicated process which involves several roles include tractor driver, boat crew and inspector. Their cooperation has a direct impact on the results of rescues which really are life and death problems. The familiarity with the operations greatly affect the speed and efficiency of the rescue. The rescuers themselves may also face severe conditions, e.g. darkness, tide, wind, and rain/snow. Fully understand and adequate practices become especially important before facing the real danger. Therefore, an advanced training process becomes vital.

Traditionally, the training procedure for novice is to have $\mathrm{him} / \mathrm{her}$ read instructional manuals and then practice in a real launching condition. However, there are almost always risks for inexperienced operators in this situation and also studying manual of a complicated operation process could be confusing for beginners to understand, especially involving operators with different roles. Luckily, the emergence of the game techniques provides us a new and effective platform to simulate the launching process which can be used for training while avoiding those risks. In this paper, we develops conceptual and technical approaches to build a 3D game platform for lifeboat launching simulator.

Game-based training for lifeboat launching confers a wide range of benefits for both academia and industry. These include the following:

1) provide an interactive and vivid virtual environment for the trainees to play, where they can experience different roles and understand the whole picture of the training process.

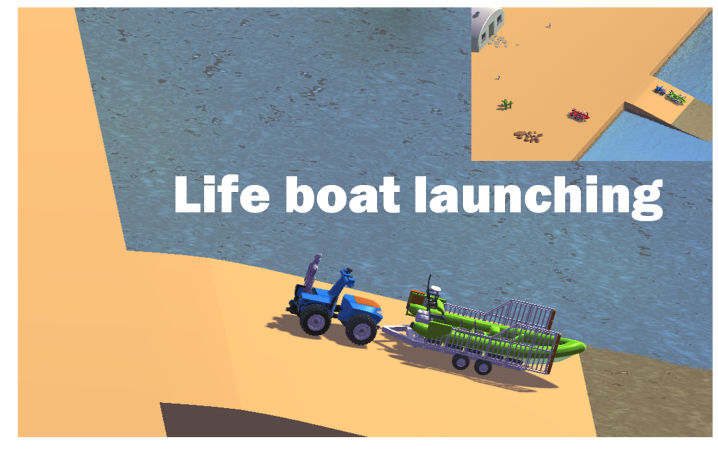

Fig. 1. Game overview

2) able to simulate dangerous or risky situations which are relatively rare in real world. It also allows the trainees to choose the training level while confident and most importantly will not risk their lives especially for novices.

3) save remarkable time and money on the training costs. Cater for remote training, trainees are not necessarily on site. It also supports peer review, feedback and training assessment.

4) ensure the learning is enjoyable where appropriate. Since the lifeboat launching process is not widely known to the public, it will also encourage public engagement for educational purpose.

Our game is based on the conjunctions of simple functional modules with each responsible for some basic task. The key modules are implemented based on physical laws which ensure the cognitive correctness for users. Since the launching process involving both on-land and in-water motions, we will discuss how different friction forces (different road condition) impact the manoeuvres of the tractor. The tractor-trailer connection also affects the behaviours of the tractor. Finally, we demonstrate how to find a proper water lever for the boat launching based on the physical fluid-solid interaction. Some of the other modules are heuristically designed. The distractions are purposely introduced to challenge and train the players' cognition of the potential hazards. The prototype gives the possibilities to adjust the function of each module easily if necessary.

We used the Unity Game Engine [1] to build up the platform. The game eventuates the performance of the players according to the scores. Finishing the tasks over the target 
time will fail the game and the algorithm penalizes players when they mess up the sequences of the training process or incur any unsafe operations, e.g., collision or turn over. More details will be demonstrated in the following sections. A good balance in the game simply is finishing the task as fast as possible with safety.

The prime objective of the present work is to design, implement, and test a complete architecture enabling the complete lifeboat launch process. In summary, this work has two main contributions:

- a novel game-based training system for beginners to be familiar with lifeboat launching process without the risks of the real world.

- a game platform to encourage public engagement for the purpose of safety education.

\section{RELATED WORK}

There is little doubt that computer games for entertainment are influencing our lives. However, it wasn't until 2003, James Gee spotlighted the many benefits of video games for learning [2].

Knowledge cannot be transmitted but is actively built. It is normally individually constructed and socially co-constructed by learners based on their interpretations of experiences in the real world. Therefore, instruction should consist of creating situations that provide interpretable experiences [3]. However, sometimes the situations are impossible in the real world for the reasons of costs, safety, time, or resource unavailability.

Luckily, the emergence of virtual reality technologies enables digital simulations of such extreme situations in which the learner can practice difficult, exacting, life-threatening, or mission-critical skills [4]. It engages through rich visuals that entice learners into fantasy worlds and at the same time permeate designed learning knowledge. However, simulations by themselves lack an intrinsic competitive element that is the hallmark of a game. Therefore, game-based learning integrated with simulation attracted the attention of researchers.

The games for educating, training and information are defined as the Serious Games [5]. Serious games allow players to experience a 'real-life' situation in an artificial environment. Many researchers and educational practitioners believe that serious games offer strong benefits in education and training through their experiential interactions [6]-[8].

Playing can be a good motivator for the learner who has to learn a skill. The entertainment dimension highlights the emotional experience, which increases the attractiveness of the game beyond its educational aim, and involves every age; for this reasons games can be considered powerful tools for training.

Game-based training gets rid of the burden of material and rests the instructors. Due to its great interactivity, this computer-based training methods make the learning process more fun and enjoyable [9]. The use of simulation systems demonstrate the possibilities for the development of different skills [10]-[13]. In the view of Gredler [6], games-based training bridged the gap between the classroom and the real world by providing experience with complex, evolving problems. Gamberini et al. [14] proved the relations between playing games and training cognitive abilities.

Fery and Ponserre analysed a golf game used to learn real launch [15]. Like virtual reality tools in general, the golf game showed a positive transfer of skills from virtual to real settings. Rosser et al. [16] emphasized how video games could be successfully used as training for laparoscopic surgeons which required high levels of visual attention, manual dexterity, and hand eye coordination. Some typical training applications of games are those targeting specific professional skills in the military personnel, such as aircraft pilots. Gopher et al. [17] observed that cadets trained with a computer game performed significantly better in flight sessions than those trained with traditional methods. As a consequence, the Israeli Air Force incorporated that game into the regular training program of its pilots. In this case the enhancement in perceptual and cognitive processing could induce significant differences in job performance. Therefore, we designed the lifeboat launching game platform for professional training, in which the key modules takes the physical laws into consideration.

Many educational simulation systems emphasize the importance of physical factors to facilitate the training process. For example, Car racing games achieve an extremely immersive user experience by means of realistic steering wheels and gear shifts paired with real-time force feedback as their preferred means of game control. Oztel and Oz [18] simulated a virtual driving platform for educational purpose which contained all necessary hardware and software modules. Such "vehicle-centred" simulators emphasized the simulation of the physical motion and sense. These simulators were designed to train learners to control the vehicle. Different from "vehicle-centred" approaches, our game is a kind of "trafficcentred" simulator, which includes but not only limited to vehicle manoeuvre. Our design is at high-level skills such as recognizing situations and developing strategies to avoid potential hazards.

Virtual environments can be also exploited in safety training in emergency situations. There exists advanced simulators for traffic safety research and driving education [19], [20]. Gamberini et al. [21] showed that in these situations users could show a change in their behaviours, in the direction of increasing the speed of their escape at the detriment of the movements' precision. In this paper, we also consider to design the game for the purpose of general public engagement. We elaborately designed and programmed series of dangerous scenarios with potential hazards during the launching process, which can subconsciously encourage the public to understand and avoid these hazards, e.g., running across the field near the launching site.

Driving simulators may consist of various pieces of equipment differing from each other. For example, some simulators are controlled with a keyboard while others with a steering wheel. Furthermore, a real car can be used as a simulator to increase the realism [19]. However, these kinds of simulation system are very expensive, the training programs involving 
these simulators are typically formal, structured, and intensive courses [22]. In this paper, we focus on presenting a low-cost 3D virtual simulation environment of lifeboat launching game using keyboard inputs, and conducting some initial evaluation results.

\section{GAME DESIGN}

In this section we demonstrate the game design which involves the pedagogical considerations. Game pipeline specifies the key operations during the lifeboat launching process. Virtual environment setting allows the players to choose the different levels according to their self-confidence.

\section{A. Game Objective and Target Users}

This game is developed to target both the professional users for lifeboat launching training and the general public for safety awareness education. The storyline is the same for both and implementations are slightly different for those two targets. Our game is consist of multiple functional modules with each responsible for a simple task. Thus the game is flexible for achieving both targets. For training purpose we focus on the correct sequence of the lifeboat launching process, while for general public engagement we emphasize on the immersion and fun of the game.

Based on the above considerations, there are several aspects we should consider in our game design, as summarized below:

\section{For training purpose:}

- the sequence of the operations becomes very important; each step of operation has to map with the instruction manual to allow fully consciousness of the operative performance;

- the training system aims to enhance the users' familiarity of the operations and improve the efficiency of the rescue under the premise of ensuring safety;

- the game also inspects the users' ability of handling hazards and emergency response;

- the game requires the understanding of the underlying physical laws behind the operations to better manipulate the vehicle.

\section{For general public:}

- the process weakens into a game that simply asks players to complete the task without the deep understanding of the process;

- the cognition of the potential hazards matters the most;

- the players doesn't need to know the exact sequence of the launching operations;

- ensure the game is enjoyable where appropriate.

\section{B. Game Pipeline and system architecture}

To allow the game serves for different purposes as discussed above, we designed the game pipeline according to the launching manual as bellow:

1) * tractor connects with the lifeboat: make sure connected properly;

2) inspector checks the environment safety and the tractor working well;
3) * tractor driver and boat crews embark;

4) tractor driver, boat crew, and inspector establish radio communications;

5) inspector gives launching signal: make sure signal received before launching;

6) * tractor moves out from the boathouse: make sure no hit or collision with environmental assets;

7) * tractor drives towards to the seaside: choose the correct driving mode according to different road conditions;

8) * tractor drives into the water: adjust the sensor, camera, light to match different environment;

9) * tractor drives to the proper launching area: find the launching area and avoid the surrounding hazards under water;

10) * tractor disconnects with lifeboat when the water depth is adequate for launching: detect the right water level;

11) * tractor drives back to the boathouse: adjust driving mode and make sure avoiding any hazards;

12) * tractor driver disembarks;

For the training purpose, all of the operations above must be completed in order. Any mistake of the sequence will receive penalty points, details in Sec. IV-D. Some operations are designed purposely for pedagogy, e.g., step 2, 4, 5, which do not associate with particular visual feedback in the game. The training instructor can set the target completion time of the game for trainees to practice and improve the launching efficiency. We designed pull-down menus for each step of the operation to emphasis the orders of operations, shown in Fig. 2.

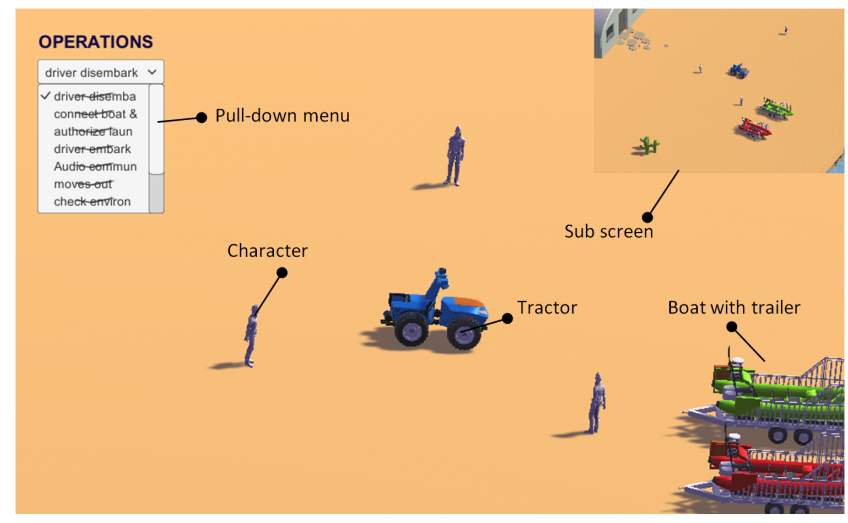

Fig. 2. System architecture

For the general public engagement, we only expect the players to fulfil the steps with * which give direct visual feedback. There will be more hazards during the launching process to make the game more entertaining, e.g., running dog, moving vehicles and pedestrians, and floating boats in water. Players should always be cautious about these hazards and move safely without incurring any danger. The check for the sequence of the operations will be disabled here. The players will be asked to release the boat at a labelled position instead of considering the water level. The players will only rely on the keyboard input without the use of pull-down menus. 
There are three main assets in this game: characters (include the tractor driver, boat crew and inspector), tractors, and boats. We use keyboard to control the motion of character, as well as the pre-recorded animation, e.g., idle, walk and embark/disembark. Once the driver embarks on the tractor, the driver is parenting with the tractor which means we ignore the relative motion of the driver and tractor. The manoeuvres of the tractor will be directly controlled using the keyboard input, same principle for the boat crew and the boat. We use a configuration joint to describe the relationship once the tractor and boat are connected, which will be detailed in Sec. IV-B. The movement of boat is trigged by tractor and effected by water. There is no external input for the motion of the boat. The relationship between them are shown in Fig. 3.

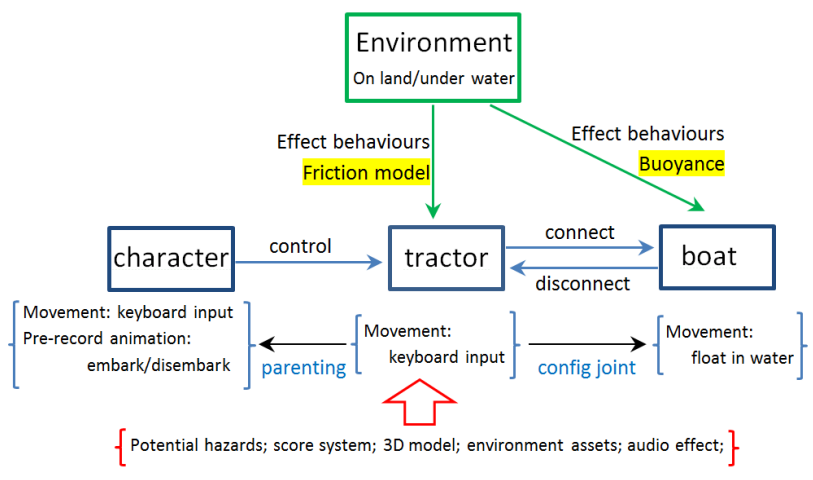

Fig. 3. System architecture

Basically the players allow manoeuvre operations to control the characters and tractors, fulfil the launching process without incur any danger within given time. The score system will give feedback at the end of the game.

\section{Virtual Environment Setting}

An individual trainee will start with standard scenarios and sufficient time. The instructor monitors the performance of the trainee. If the trainee has completed the standard scenarios without mistakes within a decent time allowance, the system will present to him scenarios with increasing difficulty. If an error is committed, feedback will be presented to the trainee, and the trainee may need to go through the same series of scenarios repeatedly until he succeeds.

We designed three levels for the game: familiar with the process; deal with hazards skilfully; handle different weather conditions.

At the final level, there will be severe weather conditions, such as rain or heavy fog which will either affect the players' visibility or increase the difficulty of the control of the tractor and boat. These weather conditions can also be superimposed or changed suddenly during the game. This is a good way to effectively exercise the trainees' response capability.

This level feature of the game allows the trainees to choose the proper levels for themselves according to their experience which avoids the situation of too difficult for novice or wasting time for experienced users.

\section{GAME IMPLEMENTATION}

In order to demonstrate the feasibility of our design, we have implemented and tested a prototype system using Unity. This prototype is completely in line with the game objectives and pipeline. We use third person view in this game since we've got more than one character for different roles in the game.

\section{A. Motion Control with Rolling Resistance}

In this game, the key motion control is the tractor manoeuvre. Presuming that the tractor provides a constant power (power $=$ force $\times$ velocity $)$ which ensures a constant forward velocity $\mathbf{v}_{0}$. Considering the constant pulling force, the speed of tractor is mainly affected by a friction force. The friction of a motor vehicle tire rolling on a road is often called rolling resistance or rolling friction. The primary cause of the rolling resistance is hysteresis: as the tire rotates under the weight of the vehicle, it experiences repeated cycles of deformation and recovery, and it dissipates the hysteresis energy loss as heat [23]. One major aspect of understanding and predicting tractor performance is that of determining the rolling resistance of a wheel.

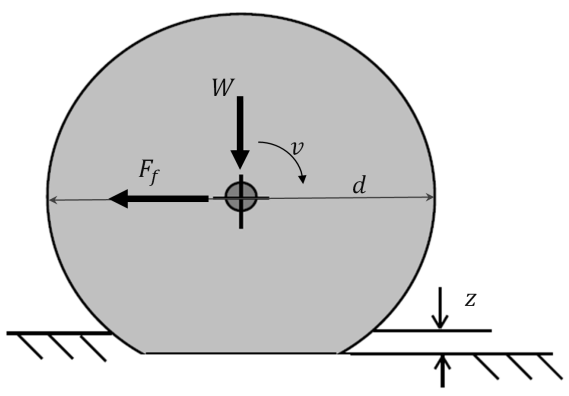

Fig. 4. Rolling resistance model.

Factors that contribute to rolling resistance are the (amount of) deformation of the wheels, the deformation of the roadbed surface, and movement below the surface. Additional contributing factors include wheel diameter, speed, load on wheel, surface adhesion, sliding, and relative micro-sliding between the surfaces of contact [24]. The problem of determining the rolling resistance, when slip (movement below the surface) is present, is more complex and will not be considered here. We apply a simple rolling resistance model here which can be calculated by

$$
\mathbf{F}_{f}=C_{r r} \mathbf{W}
$$

where $\mathbf{W}$ is the load/weight per wheel of the tractor. $C_{r r}$ is the rolling resistance coefficient. The direction of the rolling resistance force is opposing to the motion of the wheel as it rolls on a surface. For a slow rigid wheel on a perfectly elastic surface, $C_{r r}$ can be calculated by:

$$
C_{r r}=\sqrt{z / d}
$$

where $z$ is the sinkage depth and $d$ is the diameter of the rigid wheel. It is clear that the rolling resistance of a wheel 
will be a function of the strength - deformation properties of the surface and the size and deformation characteristics of the wheel.

In our game, the tractor will go through various road conditions which involves concrete road, sand, pebble way, and seawater, shown in Fig. 5. The performances of the tractor on these four terrain types are quite different. To match the speed of the tractor in simulation, we need to calculate the rolling resistance force. Therefore, it is necessary to find out the corresponding resistance coefficient $C_{r r}$ for different type of the road condition.
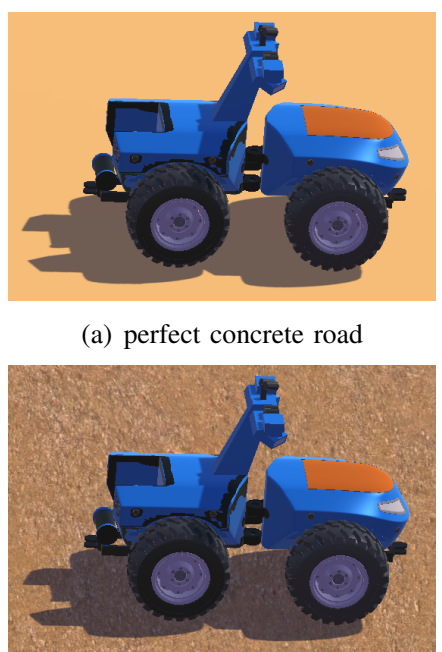

(c) pebble way (a) perfect concrete road

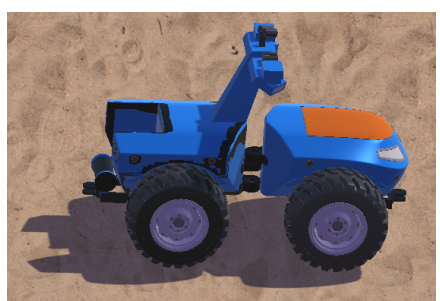

(b) sand beach

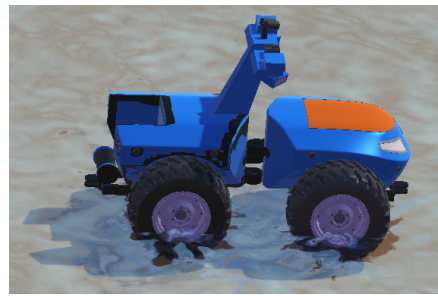

(d) submerged in seawater
Fig. 5. Various road conditions for tractor.

The $C_{r r}$ of ordinary car tires on concrete is 0.010 to 0.015 [25]. Since the tire of tractor is normally quite large, we take $C_{r r}=0.010$ for the tractor on perfect concrete road. The sand beach is quite soft and allows significant deformation. Therefore, we choose $C_{r r}=0.1$ for the sand condition [25]. The hardness of peddle way will be in-between of concrete and sand, thus we take $C_{r r}=0.0385$ [26] (alike dirt road). The tractor in this game also needs to drive down to a wet beach road. We regard the seawater as a lubricated effect on the rolling resistance force. Therefore, $C_{r r}=0.005$ for tractor submerged in seawater.

When tractor is submerged in seawater, we also need to consider the buoyancy force. The rolling resistance can then be written as:

$$
\mathbf{F}_{f}=C_{r r}\left(\mathbf{W}-\mathbf{F}_{b}\right)
$$

where $\mathbf{F}_{b}$ is the buoyancy force.

The basic manoeuvre of the vehicle includes forward, backward and turn. When the tractor is turning a angle of $\theta$ shown as Fig. 6, there will be another friction force on the vertical direction and the resultant friction force will become $\mathbf{F}_{f} / \cos \theta$. Thus, the actual turning velocity of the tractor will become $\mathbf{v}_{0} \cos \theta$, where $\mathbf{v}_{0}$ is the forward velocity.

Another situation is the tractor driving along on a slope, shown in Fig. 7. If the slope has a inclination angle $\gamma$. The

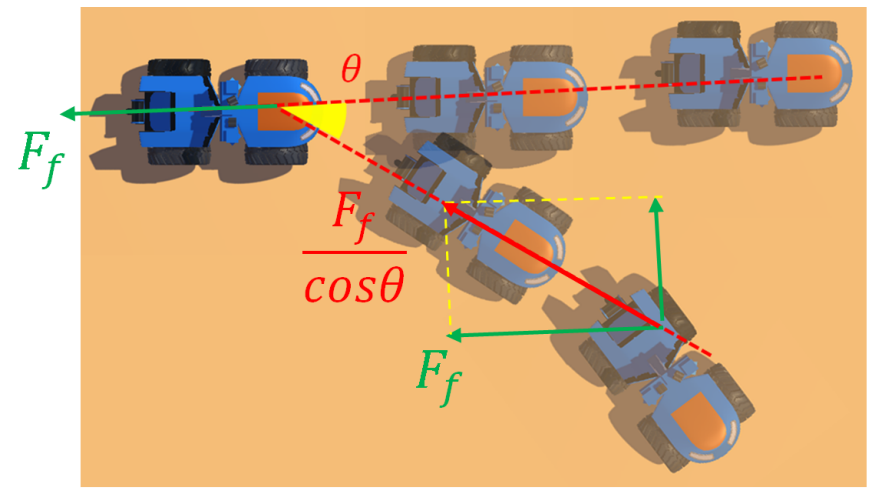

Fig. 6. Tractor turning condition.

load $\mathbf{W}$ on wheel will become $\mathbf{W} \cos \gamma$, which will decrease the rolling resistance force. The wet slope is even more slippery with a smaller $C_{r r}$.

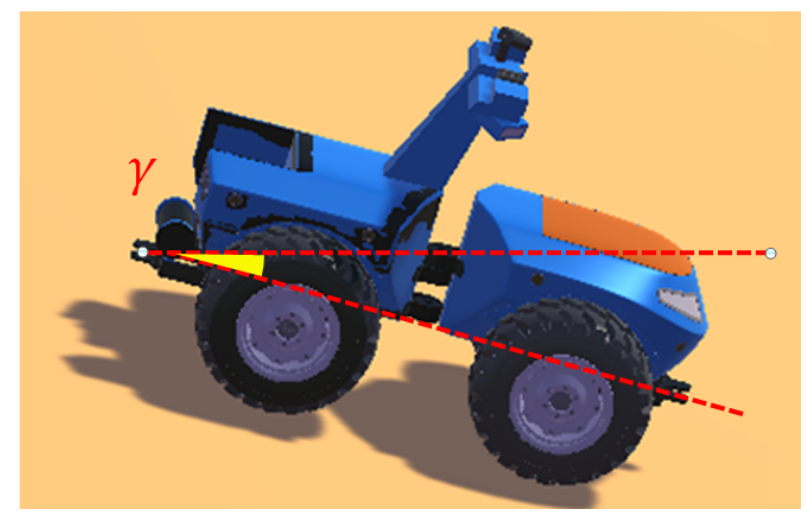

Fig. 7. Tractor on a slope.

\section{B. Tractor-trailer hitching}

In the last section, we discussed how the load of tractor determines its rolling resistance. The load depends on [27]:

1) the weight of the tractor itself;

2) part of the implement weight (if any) that is carried by the tractor;

When the tractor carries the trailer and boat, the hitching configurations on one hand limit the movement of the trailer and boat, on the other hand affect the behaviours of tractor. Tractor-trailer is a very popular hitching mode. The stability for tractor-trailer vehicle is much more complex compared with individual vehicle. However, insufficient use of the technology can cause problems such as breakdowns and failures during operations [28].

According to the tractor in real life, the pin pivot for the connection can be placed at either the front or the rear of the tractor. The connection follows the law of one point hitch. The trailer is free to move in both the horizontal and vertical planes as it follows the varying ground surface. We used a 
configuration joint for the hitching of trailer and tractor to fulfil the mechanics in both planes:

(i) the horizontal plane: the steering of the tractor has a joint effect on the turning of the trailer, shown as Fig. 8. The tractor forwards by a power-trigged drag force $\mathbf{F}_{p}$. Given the angle difference between the direction of tractor and boat $\alpha$, according to the parallelogram rule, the drag force exerting on the boat will be $\mathbf{F}_{p} \cos \alpha$. It is easy to conclude that if $\alpha$ is too big (90 degree in an extreme case), $\cos \alpha$ towards 0 , and the boat will get little drag force, leading to the difficulty of the movement. Therefore, we limit the angle $\alpha$ within 30 degree.

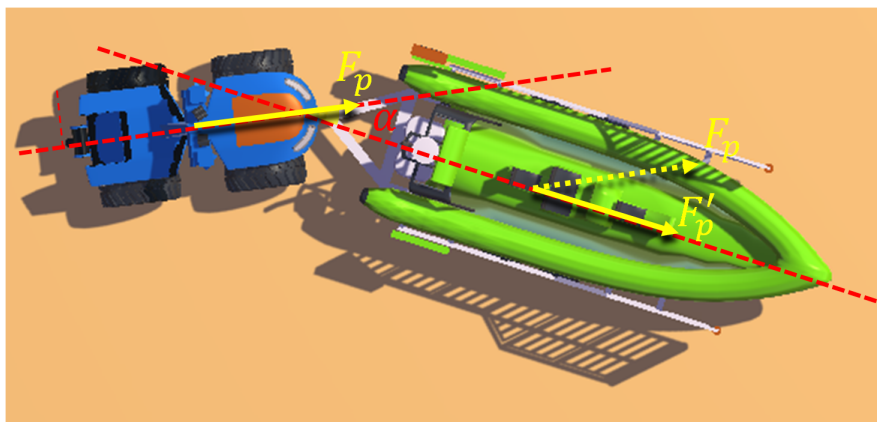

Fig. 8. Kinematic scheme of drag force, overlook plane

The resultant rolling resistance force $\mathbf{F}_{f_{t}}$ can be calculated from the resistance force of tractor $\mathbf{F}_{f_{1}}$ and the resistance force of boat $\mathbf{F}_{f_{2}}$ as Eq. 4 demonstrated in Fig. 9.

$$
\mathbf{F}_{f_{t}}=\sqrt{\mathbf{F}_{f_{1}}^{2}+\mathbf{F}_{f_{2}}^{2}+2 \mathbf{F}_{f_{1}} \mathbf{F}_{f_{2}} \cos \alpha}
$$

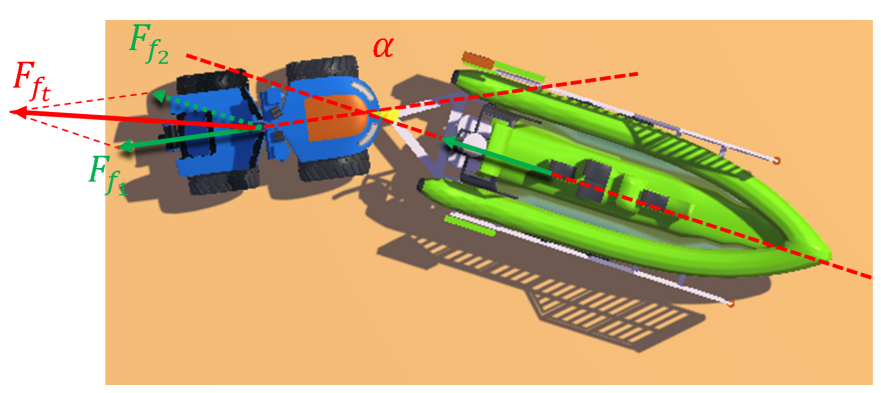

Fig. 9. Resultant of the rolling resistance forces

(ii) the vertical longitudinal plane: the tractor and trailer can have different directions on the vertical plane, e.g. on a slope, shown as Fig. 10. Due to the gravity, there will be an angle of $\beta$ between the direction of the tractor and trailer. Similar with the horizontal plane, the drag force of the boat will be $\mathbf{F}_{p} \cos \beta$. We set the limit of $\beta$ as 30 degree as well to avoid no drag force for the boat. The resultant rolling resistance force can be similarly calculated as Eq. 4.

With the connection and disconnection with the boat and trailer, the load on wheel is changing all the time. The tractor behaves differently with the changing load condition. When boat is connected, the tractor is obviously performing slower.

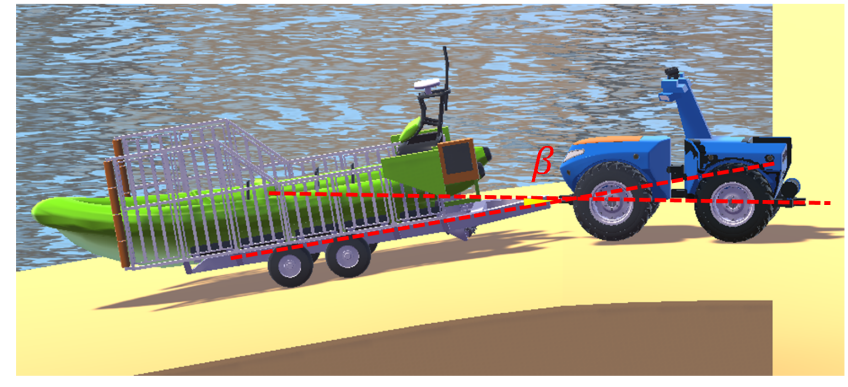

Fig. 10. Kinematic scheme, yaw plane

\section{Water Level and Buoyancy}

We calculate the buoyancy to determine the water level as the boat release criterion. Boat will float when the buoyancy $\mathbf{F}_{b}$ equals to the gravity of the boat $G$ which means:

$$
\mathbf{F}_{b}=G=\rho_{w} V^{\prime} g=\rho_{b} V g
$$

where $\rho_{w}, \rho_{b}$ are respectively the density of the water and density of the boat. $V$ is the volume the boat while $V^{\prime}$ is the submerged volume of the boat which can be written as:

$$
V^{\prime}=\frac{\rho_{b}}{\rho_{w}} V
$$

As long as the boat model is given, we can calculate the hight of the submerged volume $h$. Once the water level is larger than $h$ the boat can be released.

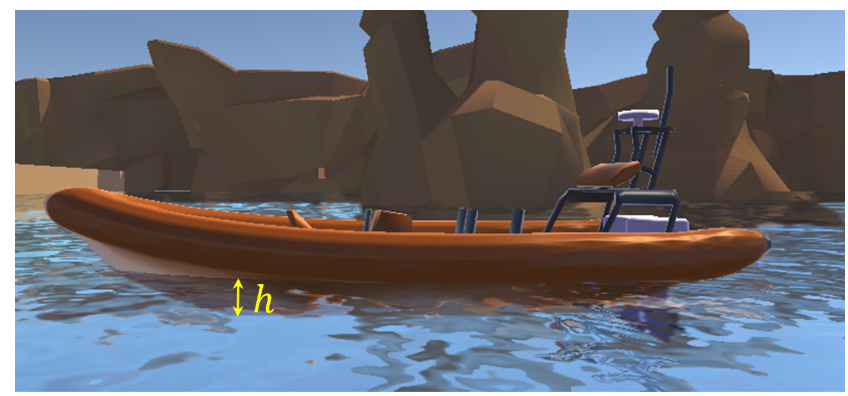

Fig. 11. water level

\section{Score System}

The full score of the system is 100. If the player runs out of the limited time, the game will fail. Each violation of incorrect operation or any incurred unsafe behaviours will trigger certain penalty points. The details of the penalty points for different violation in our scenario are shown in Table 1. Penalty points of each item can be superimposed. For example, twice collisions with the environmental assets will be 60 penalty points. As long as the score becomes negative or 0 , the game will fail and players can choose to replay.

\section{USER EVALUATION}

Our aim is to collect evidence on whether the play of the games is helping the players familiarize the lifeboat launching 


\begin{tabular}{c|c}
\hline Violations & Penalty points \\
\hline Failure to comply with the sequence of operations & 40 \\
Boat not properly connect with tractor & 40 \\
Tractor collides with environmental assets & 30 \\
Driver or crew not safely embarks/disembarks & 30 \\
Wrong driving model on different road conditions & 30 \\
Collision with human or hazards (moving vehicle, animal) & 50 \\
Tractor loses control and falls into water & 100 \\
Boat loses control or is not properly released in water & 100 \\
Boat collides with environmental assets in water & 30
\end{tabular}

TABLE I

THE GUIDE OF VIOLATIONS AND PENALTY POINTS IN OUR GAME

process and improve the safety awareness for the general public. We conduct two case studies for the two purpose of the games: one for training and the other for public awareness of the potential hazards. All of the experiments follows the principles of the research ethics policy. Written consent was obtained from each participant.

\section{Study 1: for training}

We recruited 20 volunteers (10 male, 10 female) for this case study, aged 23 to 41 . None of the participants had any life boat launching experience before. We separated the participants into two groups, for the purpose of comparative case studies. Each group has 10 participants (5 male, 5 female).

Group one was instructed to play our game. We only allow the player to fulfil one launching process in the game to keep the fairness of the comparison. Group Two was given a textual instructional manual of lifeboat launching similar with the content described in Sec. III-B. Afterwards we tested the familiarity of the lifeboat launching process by giving the participants a disrupted order of the launching operations. The participants were asked to reorder them in a limited time. The participants were tested with 5 trials, each time with a shorter allowance time. The average correctness rate of each group at each time is shown in Fig. 12.

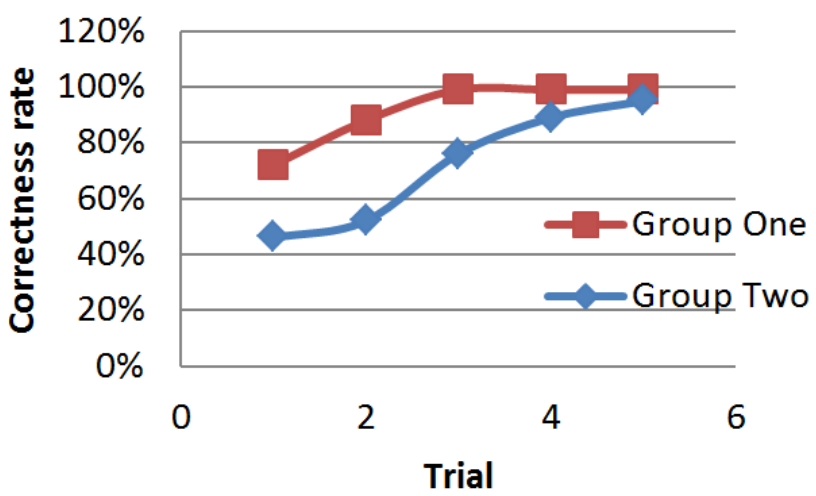

Fig. 12. Comparison of the correctness rate of two groups

The results show that the participants who played our game achieves significantly better than the one who read the manual. The participants in Group One is proven to have deeper understanding of the whole process. And they held positive attitude towards the user experience of the game. This proves that using the game to help novice to understand the launching process is more effective than reading manual. The participants in Group One enjoyed the learning experience due to the interactivity and entertaining of the game.

we also did another experiment with the same participants to gain more information about the performance of the players. All the participants were asked to play the game for 8 trials with a limited time. We tracked the average score each time. The results are plotted in Fig. 13. We found that the performance was improved rapidly and players was able to avoid more potential hazards by practice. Therefore, the idea of using the game for the professional training in the future is very promising.

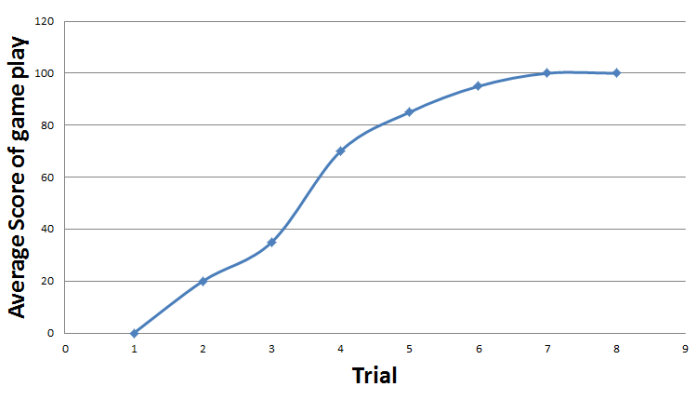

Fig. 13. Average scores in 8 games

\section{Study 2: for safety awareness}

We recruited 10 young participants ( 5 male, 5 female) for the case study, aged 6 to 14 . This study is mainly for the cognition of the potential hazards. Same with Study 1, we separate them into two groups. Group One played our game while Group Two didn't. We designed a questionnaire to test the awareness of the potential hazards during the lifeboat launching. The participants from both groups answered the same questionnaire which consists of 10 questions with the question like "What will you do when you need to cross the launching area, running across quickly or wait until the tractor passed?". We give every desired answer 10 point for each question. The average score of Group One is 78 $(100,80,80,70,60)$ while the average score of Group Two is $46(30,50,70,60,20)$ which is $41 \%$ lower.

The participants who played our game performed much better in the test of spotting and understanding the potential hazards which may happen during the life boat launching process. They also have higher possibility to pay more attention to the safety issue in the future life when they have seaside activities. Furthermore, the game also spread the knowledge of lifeboat launching.

The two studies show that the existing functions of the system have demonstrated the feasibility of our approach. The results were very promising. The game is entertaining as well as educational functioned. The system was fully functional during the test and the interactions worked smoothly. 


\section{CONCLUSION}

In this paper, we propose a game platform prototype for life boat launching process using educational and technical approaches. The game allows novices to acquire life boat launching experience in a virtual environment without the risks of the real world. It provides a much safer and more economic platform for users to get familiar with the operations. This can be used for professional training purpose in the future. Furthermore, the game serves the educational purpose of the awareness of safety issues for general public while entertaining. Games support steering tasks by providing visual feedback through images presented on a screen in front of the user. The user evaluation results prove that people learn better when they are actively engaged in acquiring and constructing knowledge in a learning-by-doing situation.

The next goal of this work is to add more tractor operations to improve the user experience. The driving model and the tractor model are graphically rather simple. Also the level of detail in the graphics and the rendering quality can still be improved. Furthermore, the potential hazards in the situations are currently limited. With sufficient physical kernels, the game can be developed as a test platform for the design of the new tractor model. This modularity of the architecture system allows us to replace or add modules in the future as a way to enhance particular features of particular situations. Currently users can only act using keyboard input and more advanced steering options can be experimented in the future.

\section{REFERENCES}

[1] W. Goldstone, Unity game development essentials. Packt Publishing Ltd, 2009.

[2] J. P. Gee, "What video games have to teach us about learning and literacy," Computers in Entertainment (CIE), vol. 1, no. 1, pp. 20-20, 2003.

[3] D. H. Jonassen, "Designing constructivist learning environments," Instructional design theories and models: A new paradigm of instructional theory, vol. 2, pp. 215-239, 1999.

[4] R. M. Epper, A. Derryberry, and S. Jackson, "Game-based learning: Developing an institutional strategy," Research Bulletin)(Louisville, CO: EDUCAUSE Center for Applied Research, 2012.

[5] C. C. Abt, Serious games. University Press of America, 1987.

[6] M. E. Gredler, "Games and simulations and their relationships to learning," Handbook of research on educational communications and technology, vol. 2, pp. 571-581, 2004.

[7] P. Backlund and M. Hendrix, "Educational games - are they worth the effort? a literature survey of the effectiveness of serious games," in Games and Virtual Worlds for Serious Applications (VS-GAMES), 2013 5th International Conference on, Sept 2013, pp. 1-8.

[8] C. Youngblut, "Educational uses of virtual reality technology." DTIC Document, Tech. Rep., 1998.

[9] F. L. Greitzer, O. A. Kuchar, and K. Huston, "Cognitive science implications for enhancing training effectiveness in a serious gaming context," Journal on Educational Resources in Computing (JERIC), vol. 7, no. 3, p. 2, 2007.

[10] J. CGM, "Research on simulator-based training and instruction strategies," Training and Simulation, p. 73, 1994.

[11] W. Schneider, "Training high-performance skills: Fallacies and guidelines," Human Factors: The Journal of the Human Factors and Ergonomics Society, vol. 27, no. 3, pp. 285-300, 1985.

[12] D. R. Michael and S. L. Chen, Serious games: Games that educate, train, and inform. Muska \& Lipman/Premier-Trade, 2005.

[13] T. Susi, M. Johannesson, and P. Backlund, "Serious games: An overview," 2007.
[14] L. Gamberini, G. Barresi, A. Maier, and F. Scarpetta, "A game a day keeps the doctor away: A short review of computer games in mental healthcare," Journal of CyberTherapy and Rehabilitation, vol. 1, no. 2, pp. 127-145, 2008.

[15] Y.-A. Fery and S. Ponserre, "Enhancing the control of force in putting by video game training," Ergonomics, vol. 44, no. 12, pp. 1025-1037, 2001.

[16] J. Rosser, P. J. Lynch, L. A. Haskamp, A. Yalif, D. A. Gentile, and L. Giammaria, "Are video game players better at laparoscopic surgical tasks," in Newport Beach, CA: Medicine Meets Virtual Reality Conference, 2004.

[17] D. Gopher, M. Well, and T. Bareket, "Transfer of skill from a computer game trainer to flight," Human Factors: The Journal of the Human Factors and Ergonomics Society, vol. 36, no. 3, pp. 387-405, 1994.

[18] I. Oztel and C. Oz, "Developing a virtual driving simulator for educational puposes," Balkan Journal of Electrical and Computer Engineering, vol. 2, no. 2, 2014.

[19] M. Lebram, "A driving simulator based on video game technology," 2006.

[20] J. Östlund, L. Nilsson, J. Törnros, and Å. Forsman, "Effects of cognitive and visual load in real and simulated driving," 2006.

[21] L. Gamberini, P. Cottone, A. Spagnolli, D. Varotto, and G. Mantovani, "Responding to a fire emergency in a virtual environment: different patterns of action for different situations," Ergonomics, 2010.

[22] P. Wolffelaar, S. Bayarri, and I. Coma, "Script-based definition of complex scenarios," in Proc. of the 4th Driving Simulation Conference, 1999, pp. 7-8.

[23] N. R. C. U. T. R. B. C. for the National Tire Efficiency Study, Tires and Passenger Vehicle Fuel Economy: Informing Consumers, Improving Performance. Transportation Research Board, 2006, vol. 286.

[24] R. Hibbeler, Engineering Mechanics: Statics \& Dynamics (Eleventh ed.). Prentice Hall, 2007.

[25] T. D. Gillespie, "Fundamentals of vehicle dynamics," SAE Technical Paper, Tech. Rep., 1992.

[26] I. O. Baker, A treatise on roads and pavements. John Wiley \& sons, Incorporated, 1919.

[27] R. H. Macmillan, "The mechanics of tractor-implement performance: theory and worked examples: a textbook for students and engineers," 2002.

[28] S. Aloni and S. Khedkar, "Comparative evaluation of tractor trolley axle by using finite element analysis approach," International Journal of Engineering Science and Technology (IJEST). Vol4, no. 4, 2012. 